\title{
A graphene electron lens
}

\author{
L. Gerhard, ${ }^{1}$ E. Moyen, ${ }^{2}$ T. Balashov, ${ }^{1}$ I. Ozerov, ${ }^{2}$ M. Portail, ${ }^{3}$ H. Sahaf,${ }^{2}$ L. Masson, ${ }^{2}$ \\ W. Wulfhekel, ${ }^{1}$ and M. Hanbücken ${ }^{2}$ \\ ${ }^{1}$ Physikalisches Institut, Karlsruhe Institute of Technology, Wolfgang-Gaede-Str. 1, 76131 Karlsruhe, \\ Germany \\ ${ }^{2}$ CINaM-CNRS, Aix-Marseille University, Campus Luminy - Case 913, 18288 Marseille, France \\ ${ }^{3}$ CRHEA-CNRS, Parc de Sophia - Antipolis, rue B. Gregory, 06560 Valbonne, France
}

(Received 26 November 2011; accepted 20 March 2012; published online 10 April 2012)

\begin{abstract}
An epitaxial layer of graphene was grown on a pre patterned $6 \mathrm{H}-\mathrm{SiC}(0001)$ crystal. The graphene smoothly covers the hexagonal nano-holes in the substrate without the introduction of small angle grain boundaries or dislocations. This is achieved by an elastic deformation of the graphene by $\approx 0.3 \%$ in accordance to its large elastic strain limit. This elastic stretching of the graphene leads to a modification of the band structure and to a local lowering of the electron group velocity of the graphene. We propose to use this effect to focus two-dimensional electrons in analogy to simple optical lenses. (C) 2012 American Institute of Physics. [http://dx.doi.org/10.1063/1.3701594]
\end{abstract}

Two dimensional $\mathrm{sp}_{2}$ carbon, i.e., graphene, is a versatile and promising material for future high speed electronics. ${ }^{1}$ It combines an extremely high electron mobility with a unique band structure resembling that of the photon ${ }^{2,3}$ such that the electrons in graphene can be considered as massless Dirac fermions. ${ }^{4,5}$ Even impurities disturbing to the local electronic structure do not lead to scattering due to Klein tunneling. ${ }^{6}$

Graphene can be prepared either by exfoliation ${ }^{4}$ from graphite or more reproducibly by growth on suitable substrates such as $\mathrm{SiC}(0001) .^{7}$ In the latter case, high quality graphene films could be achieved, ${ }^{8}$ and due to the large band gap of $\mathrm{SiC}$, the graphene layer is basically decoupled from the bulk states of the substrate. ${ }^{9}$

There are different approaches to pattern graphene as to build electronic devices. For example, nano-ribbons of graphene can be used to build high mobility transistors, ${ }^{9}$ or by using pn-junctions as substrates for graphene, electron lenses can be realized. ${ }^{10}$ Recently, it has been shown by ab-initio calculations that straining graphene leads to a slight modification of the band structure and as a consequence to a change of the Fermi velocity. ${ }^{11-13}$ This offers a way to design graphene-based device. Especially, by locally changing the strain, it was proposed that nano-channels can be formed.

In this letter, we show that smooth but locally strained layers of graphene can be grown on pre-patterned $6 \mathrm{H}$ SiC(0001) samples. This should lead to devices similar to optical lenses, i.e., these devices can be used to focus plane electron waves in two dimensions to a focal point. This is a simple alternative to using pn-junctions ${ }^{10}$ as focusing elements.

The sample preparation included several steps. In a first step, porous $\mathrm{Al}_{2} \mathrm{O}_{3}$ membranes were fabricated by hard anodic oxidation of aluminum. ${ }^{14,15}$ Two inches Al $(99.999 \%)$ discs were electropolished in perchloric acid:ethanol (1:4) at $20 \mathrm{~V}$ for several minutes. Porous alumina membranes with a pore diameter of $\approx 50 \mathrm{~nm}$ and a lattice constant of about $300 \mathrm{~nm}$ were then grown in $0.015 \mathrm{M} \mathrm{H}_{2} \mathrm{C}_{2} \mathrm{O}_{4}$ by applying $140 \mathrm{~V}$. A first $50 \mu \mathrm{m}$ thick alumina layer was formed after $6 \mathrm{~h}$ and was then removed by chemical dissolution with chromic acid. The resulting imprinted $\mathrm{Al}$ surface was then ano- dized a second time for $30 \mathrm{~s}$ leading to a $1 \mu \mathrm{m}$ thick membrane. Free-standing porous alumina membranes were then obtained by dissolving the $\mathrm{Al}$ substrate in a $\mathrm{CuSO}_{4}: \mathrm{HCl}: \mathrm{H}_{2} \mathrm{O}(2: 1: 10)$ mixture. The pores were subsequently opened by thinning the barrier layer through reactive ion etching using a mixture of $\mathrm{SF}_{6}: \mathrm{O}_{2}(4: 1)$ for $2 \mathrm{~min}$ at 10 mbar and $150 \mathrm{~W}$.

In a second step, the alumina membrane was placed onto epiready $6 \mathrm{H}-\mathrm{SiC}(0001)$ wafers, and the pores were transferred as holes to the $\mathrm{SiC}$ sample by reactive ion etching using the same mixture as for pore opening for $10 \mathrm{~min}$ (see Fig. 1(a)). The residual membrane was then removed by chemical etching in $30 \% \mathrm{H}_{3} \mathrm{PO}_{4}$ solution for $30 \mathrm{~min}$ at $45^{\circ} \mathrm{C}$. The patterned $\mathrm{SiC}$ samples were subsequently etched at $1550{ }^{\circ} \mathrm{C}$ under $\mathrm{H}_{2}$ for $30 \mathrm{~s}$ in a hot wall reactor to achieve a reorganization of the etch pits to regular and hexagonal holes (see Fig. 1(a)). ${ }^{16}$ As the multi-step sample preparation is a massively parallel process, a large number of holes were created in $\mathrm{SiC}$. The patterned $\mathrm{SiC}$ samples were finally investigated with scanning electron microcopy (SEM) and atomic force microscopy (AFM). The SEM image of Fig. 1(b) shows the overall structure of regular array of hexagonal holes with a pitch of $\approx 300 \mathrm{~nm}$ and a small spread in hole diameter. The well ordered shape reflects the minimization of the surface free energy due to the Wulff construction. ${ }^{17}$

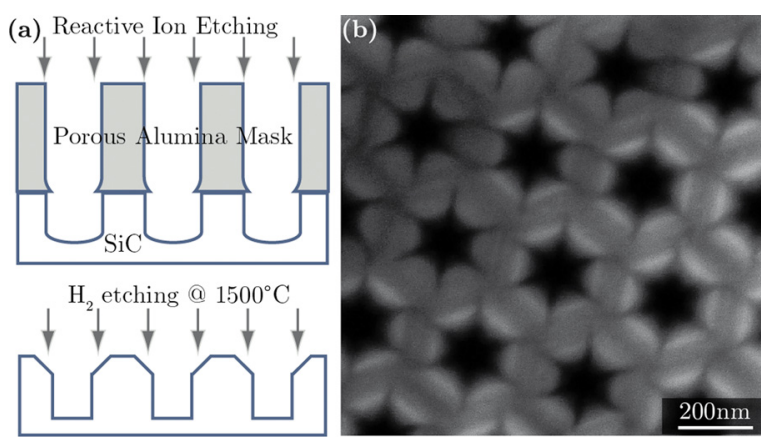

FIG. 1. (a) Schematics of the patterning process of $\mathrm{SiC}(0001)$. Nano-holes are created on the $\mathrm{SiC}(0001)$ surface through a porous alumina mask. The wafer is then etched in hydrogen to form regular nano-holes of hexagonal shape. (b) SEM image of the patterned SiC(0001) wafer after hydrogen etching showing regular hexagonal holes with well defined facets. 
AFM (not shown) revealed inclinations of the six facets of the pores between 5 and $7^{\circ}$ to the surface plane and depth between 2 and $12 \mathrm{~nm}$.

In a third step, the patterned $\mathrm{SiC}$ samples were transferred into ultra high vacuum and were heated to $1200^{\circ} \mathrm{C}$ at low pressure $\left(\leq 10^{-7} \mathrm{mbar}\right)$ for $20 \mathrm{~min}$ by direct heating. This heating leads to the selective sublimation of Si such that the surface is enriched with C (Ref. 7), and finally few layers of graphene are formed on the substrate. ${ }^{18}$ The samples were then transferred into a room temperature scanning tunneling microscope (STM) within the same ultra high vacuum (UHV) apparatus, and the surface was imaged with atomic resolution.

The large scale image (see Fig. 2(a)) shows the regular, hexagonal shape of the hole. It consists of concentric steps of a height of 0.75 and $1.5 \mathrm{~nm}$ reflecting the step height in $6 \mathrm{H}-\mathrm{SiC}(0001) .{ }^{19,20}$ The hole is about $6 \mathrm{~nm}$ deep with facets declined by $\approx 6^{\circ}$ from the surface plane and a flat, hexagonal bottom (see also line section). Across the complete hole, a continuous film of few layers of graphene is present that smoothly follows the topography of the hole. On the flat areas, the graphene shows a weak $(6 \times 6)$ superstructure (see right inset) indicating that the film consists of 2-3 monolayers (ML) of graphene. ${ }^{18,21}$ The $(6 \times 6)$ superstructure does not affect the particular band structure of graphene as has been observed by angle resolved photoemission. ${ }^{22}$ Within the hole a continuous $(1 \times 1)$ structure of the graphene in the large scan is indicative for an undisturbed layer of 2-3 ML of graphene. Higher resolution scans were taken in the indicated areas (c-j) and are shown in higher magnification. In all areas, the same $(1 \times 1)$ structure with the same lattice orientation was found. The Fourier transform of the dashed box of Fig. 2(a) is shown in the inset. It yields six spots reflecting the hexagonal super structure of graphene on $\mathrm{SiC}$. Even in the corners of the holes, where two or three facets meet, the atomic arrangement of graphene is undisturbed (see scan j). Thus, the graphene layer adopts the shape of the hole without lattice defects but by a slight elastic deformation. From the cross section of the hole, an average tensile strain of $\approx 0.28 \%$ of the graphene layer in the hole with respect to the areas outside the hole can be estimated. This seemingly large deformation is well below the elastic limits of graphene of up to $30 \% .^{13}$ The strain equals to a stress of 3-4 GPa, which is substantial but well below the maximal values for graphene of $1 \mathrm{TPa}$. In the local area of the sample investigated with STM, the facet inclination of different holes varied between $5^{\circ}$ and $7^{\circ}$ and the hole depth between 2 and $12 \mathrm{~nm}$. These represent a strain of $0.09 \%-0.7 \%$ in the graphene layer.

Thus, the graphene layer is locally stretched and expanded. This expansion in turn is expected to affect the band structure of graphene. As recent calculations have shown, ${ }^{11-13}$ an isotropic tensile strain of graphene leads to a flattening of the bands and thus to a lowering of the group velocities by $1.5 \%$ per $1 \%$ of strain. ${ }^{13}$ This effect together with the longer geometrical path across the hole should therefore lead to a retardation of electrons traveling across the hole in comparison to electrons traveling in flat regions of the graphene. The total delay of the electrons amounts to $\approx 0.7 \%$ of the traveling time across the hole depicted in Fig.
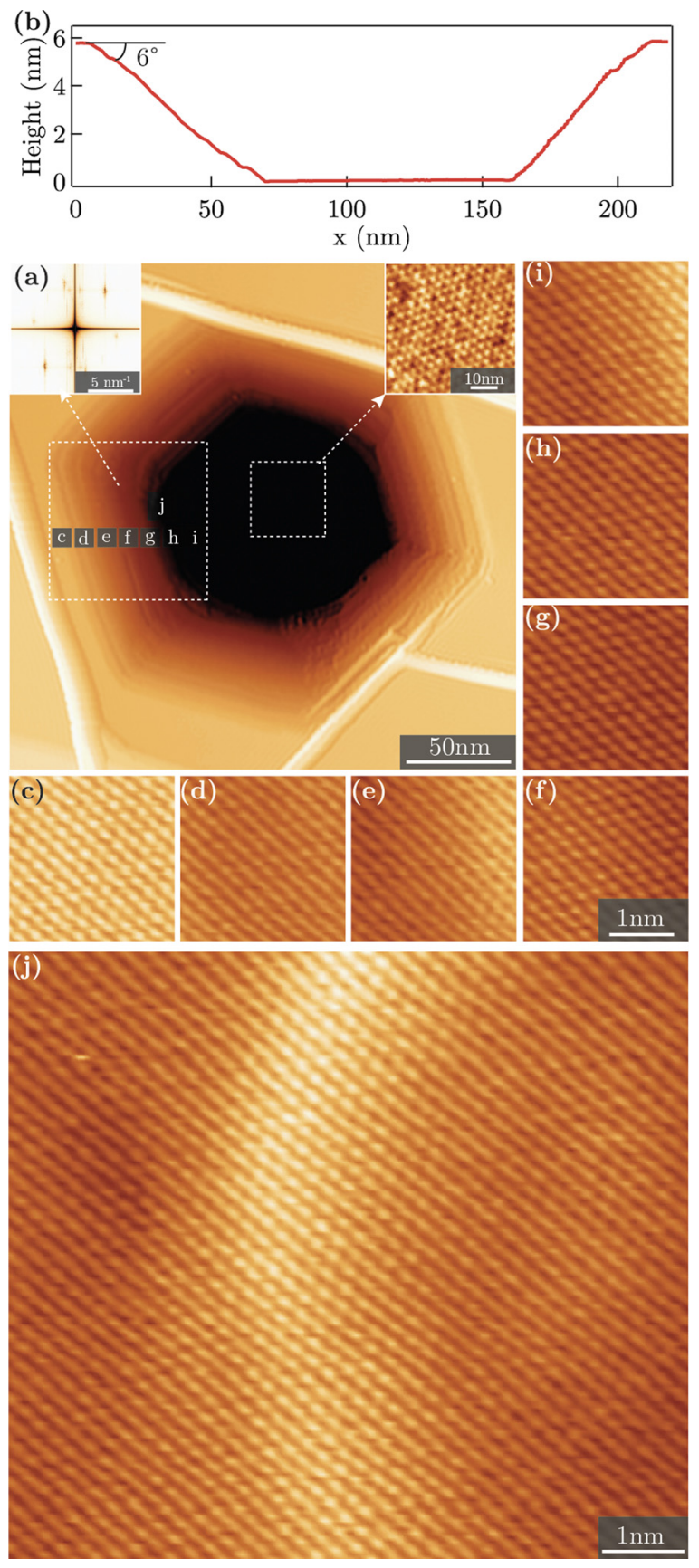

FIG. 2. (a) Large scale STM image of one nano-hole in the graphenized $\mathrm{SiC}(0001)$ wafer. The left inset shows the Fourier transform of the area in the dotted box. It displays a sixfold pattern caused by the mono-domain $(1 \times 1)$ structure of the few layer graphene. The right inset shows a low pass filtered image of the structure at the bottom of the hexagonal hole and reveals a $(6 \times 6)$ superstructure of the few layer graphene film. (b) Line section of the hole $(c-j)$ show STM images of higher resolution at places indicated in (a). The $(1 \times 1)$ structure is clearly visible showing no dislocations or small angle domain boundaries even in the corner of the holes (j).

2(a). This delay in turn should act on a plane electron wave as a focusing lens in full analogy to an optical lens of high refractive index. From the geometry of the hole and the estimated delay, an easy calculation gives a focal length of $3600 \mathrm{~nm}$. We note that the graphene layer is also stressed by the different thermal expansion coefficients of graphene and the substrate. ${ }^{23}$ This effect, however, does not alter the lens properties, as the group velocity of the plane and the hole 


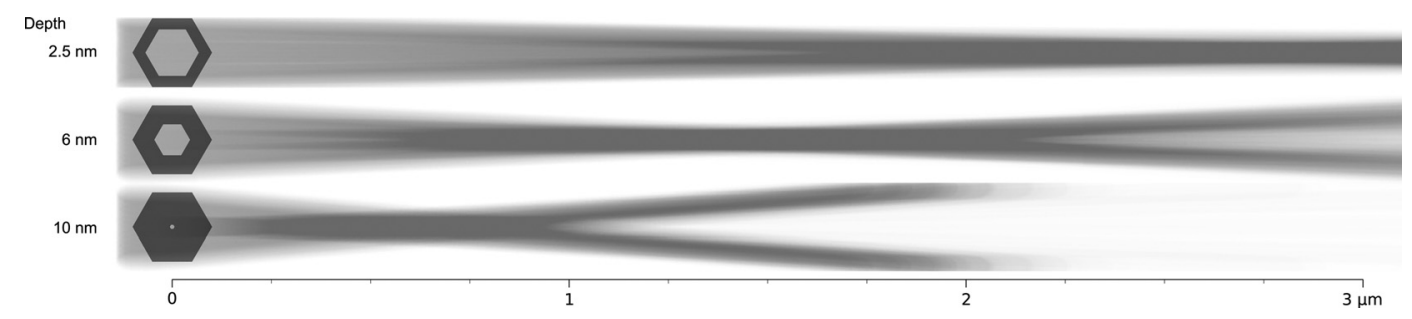

FIG. 3. Calculated electron propagation through strained graphene lenses. All three lenses have the same outer diameter of $200 \mathrm{~nm}$ with varying depth as indicated in the figure. The side facets are inlined by 5,6 and $7^{\circ}$ for the holes of $2.5,6$, and $10 \mathrm{~nm}$ depth, respectively.

areas are modified through thermal expansion by the same amount.

For a more accurate estimation, we have numerically calculated the propagation of electrons through several lenses, taking into account the anisotropic strain on the facets of the nano-hole (see Fig. 3)). The stress, with it the electron group velocities, was considered to be constant along the slope and raising linearly with depth perpendicular to the slope. The calculation was done using the Lagrangian formalism for minimization of the optical path in an anisotropic inhomogeneous media. ${ }^{24}$ Only refraction was considered and diffraction was neglected, as the lens diameter is much larger than the Fermi wavelength of the electrons. The results show that the different lenses have focal regions depending on their geometry. For an intermediate hole depth of $6 \mathrm{~nm}$, the focal region is about $500 \mathrm{~nm}$ wide with the focal spot about $1.8 \mu \mathrm{m}$ away from the center of the lens. Deeper holes display a sharper focus closer to the lens and shallower holes, a less well defined focus at focal length beyond $1.8 \mu \mathrm{m}$.

A further effect that might affect the focusing properties of the lens, but was not taken into account in the calculations, is the local deformation of the graphene from its plane configuration at the edges of the hole and of the inclined planes that alters the position of the Fermi level. ${ }^{25}$ This effect can contribute slightly to further delay the electrons leading to shorter focal lengths.

In conclusion, we have investigated elastically strained graphene layers on patterned $\mathrm{SiC}(0001)$ surfaces. The graphene layer, which smoothly covers the hexagonal nanoholes of the substrate, is thus locally strained. The tensile strain in turn modifies the graphene band structure and leads to a lowering of the group velocities. This way, the structure acts as a two-dimensional electron lens in analogy to an optical lens of a higher refractive index than the surrounding. These lenses can potentially be used as focusing elements for the electrons in the two-dimensional electron gas of graphene, opening the possibility to deliberately design electron optics in two dimensions by pre patterning $\mathrm{SiC}$ substrates.
This work is dedicated to the late Ulrich Gösele, former director at the MPI of Microstructure Physics, Halle, Germany, who introduced us to the beauty and usefulness of alumina membranes. This work was funded through the French Foreign Ministry (PAI-PROCOPE No. 07639QK) and the DAAD/Procope. Fruitful discussions with F. Evers and R. Danneau are acknowledged.

\footnotetext{
${ }^{1}$ A. Geim and K. Novoselov, Nat. Mater. 6, 183 (2007).

${ }^{2}$ K. Novoselov, A. Geim, S. Morozov, D. Jiang, M. Katsnelson, I. Grigorieva, S. Dubonos, and A. Firsov, Nature 438, 197 (2005).

${ }^{3}$ Y. Zhang, Y. Tan, H. Stormer, and P. Kim, Nature 438, 201 (2005).

${ }^{4}$ K. Novoselov, A. Geim, S. Morozov, D. Jiang, Y. Zhang, S. Dubonos, I. Grigorieva, and A. Firsov, Science 306, 666 (2004).

${ }^{5}$ J. Sloczewski and P. Weiss, Phys. Rev. 109, 272 (1958).

${ }^{6}$ N. Peres, Rev. Mod. Phys. 82, 2673 (2010).

${ }^{7}$ A. V. Bommel, J. Crombeen, and A. V. Tooren, Surf. Sci. 48, 463 (1975).

${ }^{8}$ C. Berger, Z. Song, T. Li, X. Li, A. Ogbazghi, R. Feng, Z. Dai, A. Marchenkov, E. Conrad, P. First et al., J. Phys. Chem. B 108, 19912 (2004).

${ }^{9}$ C. Berger, Z. Song, X. Li, X. Wu, N. Brown, C. Naud, D. Mayou, T. Li, J. Hass, A. Marchenkov et al., Science 312, 1191 (2006).

${ }^{10}$ V. Cheianov, V. Falkon, and B. Altshuler, Science 315, 1252 (2007).

${ }^{11}$ M. Mohr, K. Papagelis, J. Maultzsch, and C. Thomsen, Phys. Rev. B 80, 205410 (2009).

${ }^{12}$ F. M. D. Pellegrino, G. G. N. Angilella, and R. Pucci, Phys. Rev. B 81, 035411 (2010).

${ }^{13}$ S.-M. Choi, S.-H. Jhi, and Y.-W. Son, Phys. Rev. B 81, 081407R (2010).

${ }^{14} \mathrm{H}$. Masuda and K. Fukuda, Science 268, 1466 (1995).

${ }^{15}$ W. Lee, U. G. R. Ji, and K. Nielsch, Nat. Mater. 5, 741 (2006).

${ }^{16}$ A. Leycuras, Mater. Sci. Forum 241, 338 (2000).

${ }^{17}$ G. Wulff, Z. Krystallogr. Mineral. 34, 449 (1901).

${ }^{18}$ P. Lauffer, K. Emtsev, R. Graupner, T. Seyller, and L. Ley, Phys. Rev. B 77, 155426 (2008).

${ }^{19}$ W. Wulfhekel, D. Sander, S. Nietsche, F. Dulot, A. Leycuras, and M. Hanbücken, Surf. Sci. 550, 8 (2004).

${ }^{20}$ F. Dulot, L. Mansour, A. Leycuras, W. Wulfhekel, D. Sander, F. A. d'Avitaya, and M. Hanbücken, Appl. Surf. Sci. 187, 319 (2002).

${ }^{21}$ C. Ridel, U. Starke, J. Bernhardt, M. Franke, and K. Heinz, Phys. Rev. B 76, 245406 (2007).

${ }^{22}$ A. Bostwick, T. Ohta, T. Seyller, K. Horn, and E. Rotenberg, Nat. Phys. 3, 36 (2007).

${ }^{23}$ K. S. Kim, Y. Zhao, H. Jang, S. Lee, J. Kim, K. Kim, J.-H. Ahn, P. Kim, J.-Y. Choi, and B. Hong, Nature 457, 706 (2009).

${ }^{24}$ A. Joets and R. Ribotta, Opt. Commun. 107, 200 (1994).

${ }^{25}$ S. Bero, A. Arnold, F. Evers, R. Narayanan, and P. Wölfle, Phys. Rev. B 82, 195445 (2010).
} 
Applied Physics Letters is copyrighted by the American Institute of Physics (AIP). Redistribution of journal material is subject to the AIP online journal license and/or AIP copyright. For more information, see http://ojps.aip.org/aplo/aplcr.jsp 